\title{
Rotation invariant moment problems
}

\author{
by \\ CHRISTIAN BERG \\ and \\ MARCO THILL \\ University of Copenhagen \\ Copenhagen, Denmark \\ University of Copenhagen
Copenhagen, Denmark
}

\section{Introduction}

An important theorem of Marcel Riesz, cf. [14], states that the polynomials are dense in $L^{2}(\mu)$, when $\mu$ is a determinate measure on the real line. In the indeterminate case Riesz also characterized the measures $\mu$ for which the polynomials are dense in $L^{2}(\mu)$. They are the so-called Nevanlinna extremal measures, introduced in Nevanlinna [11].

It does not seem to be known whether the polynomials are dense in $L^{2}(\mu)$, when $\mu$ is a determinate measure on $\mathbf{R}^{d}, d>1$, cf. the expository paper by Fuglede [7], as well as the research problems book [8, p. 529], where Devinatz poses the problem as question 1 and ascribes it to the physicist John Challifour (1978).

In this paper we shall settle the question in the negative. There exist rotation invariant measures $\mu$ on $\mathbf{R}^{d}, d>1$, which are determinate but for which the polynomials are not dense in $L^{2}(\mu)$. Such measures $\mu$ are necessarily of the following very special form

$$
\mu=\sum_{n=0}^{\infty} \alpha_{n} \omega_{r_{n}}
$$

where $\alpha_{n}>0, \omega_{r}$ is the normalized uniform distribution on the sphere $\|x\|=r$ and $0 \leqslant r_{0}<r_{1}<\ldots \rightarrow \infty$ are the zeros of an entire function of order $\leqslant 2$. Thus, if a rotation invariant and determinate measure $\mu$ does not have this form, in particular if $\mu$ has a density with respect to Lebesgue measure on $\mathbf{R}^{d}$, then the polynomials are dense in $L^{2}(\mu)$.

The above result is obtained by studying the relation between a rotation invariant measure $\mu$ and its image $\sigma$ under the mapping $x \mapsto\|x\|^{2}$ of $\mathbf{R}^{d}$ onto [0, [. Our main results are Theorems 3.2 and 3.6 from section 3 below. They contain the following results:

(1) $\mu$ is determinate if and only if $\sigma$ is determinate in the sense of Stieltjes. 
(2) The polynomials in $d>1$ variables are dense in $L^{2}(\mu)$ if and only if the polynomials in one variable are dense in $L^{2}\left(t^{k} d \sigma(t)\right)$ for any integer $k \geqslant 0$.

The result corresponding to (2) in one dimension reads as follows (where $\mu$ is symmetric and $\sigma$ is the image under $x \mapsto x^{2}$ ):

(3) The polynomials in one variable are dense in $L^{2}(\mu)$ if and only if the polynomials in one variable are dense in $L^{2}(\sigma)$ and in $L^{2}(t d \sigma(t))$.

The difference in the nature of the results in (2) and (3) stems from the theory of spherical harmonics. In dimension one there exist homogeneous harmonic polynomials of degrees 0 and 1 only, but in higher dimensions there exist homogeneous harmonic polynomials of any degree.

The existence of a rotation invariant measure $\mu$ which is determinate, but for which the polynomials are not dense in $L^{2}(\mu)$, follows, if we construct a measure $\sigma$ on [0, [ which is determinate in the sense of Stieltjes, but for which the polynomials are not dense in $L^{2}\left(t^{k} d \sigma(t)\right)$ for some integer $k$ (necessarily $\geqslant 3$ ). In sections 4 and 5 we show how to construct and characterize such measures $\sigma$.

Despite the solution in the negative of the Challifour-Devinatz problem, it turns out that the polynomials are dense in $L^{p}(\mu)$ for any $p \in[1,2[$, when $\mu$ is determinate and rotation invariant, cf. Corollary 3.10 .

If the polynomials are not dense in $L^{2}(\mu)$ for some rotation invariant measure $\mu$, then they are far from being dense in the sense that the orthogonal complement of the polynomials is of infinite dimension, cf. Theorem 4.3.

In section 1 we have put together some basic facts about measures and moments.

Section 2 treats rotation invariant measures, and we give necessary and sufficient conditions for a sequence $s: \mathbf{N}^{d} \rightarrow \mathbf{R}$ to be the moment sequence of a rotation invariant measure. For a rotation invariant measure $\mu$ on $\mathbf{R}^{d}, d>1$, we present a method of describing a canonical family of orthogonal polynomials with respect to $\mu$ in terms of the orthogonal polynomials with respect to each of the measures $t^{k} d \sigma(t)$, where $\sigma$ is the image measure of $\mu$ under $x \mapsto\|x\|^{2}$ and $k$ is any integer $\geqslant 0$.

Section 3 contains the main results as described above.

In [7] Fuglede introduced the notions of strong determinacy and ultradeterminacy. A determinate measure $\mu$ for which the polynomials are not dense in $L^{2}(\mu)$ is not strongly determinate, thereby showing that strong determinacy is a de facto stronger condition than determinacy. In [15] Schmüdgen gives another example of a determinate measure which is not strongly determinate, and he also gives an example of a strongly determinate measure which is not ultradeterminate. 
That ultradeterminacy is de facto stronger than strong determinacy is not demonstrated in this paper. We prove on the contrary that the two concepts coincide within the class of rotation invariant measures with the concept of those measures $\mu$ for which the polynomials are dense in $L^{2}(\mu)$. These questions together with a condition of Nelson, cf. [10], are discussed in section 6 , which also contains some examples.

\section{Preliminaries about measures and moments}

For a locally compact space $X$ we denote by $C_{\mathrm{c}}(X)$ the set of continuous functions $f: X \rightarrow \mathrm{C}$ with compact support and by $M(X)$ the set of positive Radon measures on $X$. We shall equip $M(X)$ with the vague topology, i.e., the weakest topology in which the evaluations $\mu \mapsto \int f d \mu$ at functions $f \in C_{\mathrm{c}}(X)$ are continuous.

If $X, Y$ are locally compact spaces and $j: X \rightarrow Y$ is continuous, we can define the (Radon) image measure $\mu^{j} \in M(Y)$ of a Radon measure $\mu$ on $X$ provided that $j$ is proper in the sense that $j^{-1}(K)$ is compact in $X$ for any compact set $K \subseteq Y$. The image measure is characterized by the equations

$$
\int f d \mu^{j}=\int f \circ j d \mu \text { for all } f \in C_{\mathrm{c}}(Y) .
$$

Let $\mathscr{M}^{*}\left(\mathbf{R}^{d}\right)$ denote the set of all positive Radon measures $\mu$ on Euclidean space $\mathbf{R}^{d}$ having moments of every order, meaning that $x^{\alpha} \in L^{1}(\mu)$ for every $\alpha \in \mathbf{N}^{d}$, where we use the multi-index notation $x^{\alpha}=x_{1}^{\alpha_{1}} \ldots x_{d}^{\alpha_{d}}$ for $x=\left(x_{1}, \ldots, x_{d}\right) \in \mathbf{R}^{d}, \alpha=\left(\alpha_{1}, \ldots, \alpha_{d}\right) \in \mathbf{N}^{d}$, $\mathbf{N}=\{0,1, \ldots\}$. As usual we put $|\alpha|=\alpha_{1}+\ldots+\alpha_{d}$.

For $\mu \in \mathscr{M}^{*}\left(\mathbf{R}^{d}\right)$ the corresponding moment sequence $s=s_{\mu}: \mathbf{N}^{d} \rightarrow \mathbf{R}$ is defined by

$$
s(\alpha)=s_{\mu}(\alpha)=\int_{\mathbf{R}^{d}} x^{\alpha} d \mu(x), \quad \alpha \in \mathbf{N}^{d} .
$$

We define $\mu, \nu \in \mathcal{M}^{*}\left(\mathbf{R}^{d}\right)$ to be equivalent, denoted $\mu \sim \nu$, if $s_{\mu}(a)=s_{\nu}(\alpha)$ for all $\alpha \in \mathbf{N}^{d}$. The equivalence class $[\mu]$ consisting of all $\nu \in \mathscr{M}^{*}(\mathbf{R})$ having the same moments as $\mu$ is a convex set, which is compact in the vague topology, cf. [3, Proposition 6.1.7].

A measure $\mu \in \mathcal{M}^{*}\left(\mathbf{R}^{d}\right)$ or its moment sequence $s$ is said to be determinate if $[\mu]$ reduces to a single measure, and indeterminate if this is not the case.

We define

$$
\mathcal{M}^{*}\left(\left[0, \infty[)=\left\{\sigma \in \mathcal{M}^{*}(\mathbf{R}) \mid \operatorname{supp}(\sigma) \subseteq[0, \infty[\} .\right.\right.\right.
$$

For $\sigma \in \mathcal{M}^{*}([0, \infty[)$ the corresponding moment sequence $s$ is called a Stieltjes moment 
sequence. We say that $\sigma$ or $s$ is determinate in the sense of Stieltjes (short: $\operatorname{det}(\mathrm{S})$ ) if $\sigma$ is the only measure on $[0, \infty$ [ with the same moments as $\sigma$, and we say that $\sigma$ or $s$ is indeterminate in the sense of Stieltjes (short: indet(S)) if there exist different measures on $[0, \infty[$ with the same moments as $\sigma$. Clearly, if $\sigma$ is indet(S) then $\sigma$ is indeterminate (sometimes called indeterminate in the sense of Hamburger). It can happen that $\sigma$ is indeterminate and yet determinate in the sense of Stieltjes, cf. [1, p. 240].

If $\mu \in \mathcal{M}^{*}(\mathbf{R})$ is indeterminate, then it is elementary to see that $t^{2} d \mu(t)$ is again indeterminate. Similarly, if $\sigma \in \mathscr{M}^{*}([0, \infty[)$ is indet(S), then $t d \sigma(t)$ is indet(S).

For $\mu \in \mathscr{M}^{*}\left(\mathbf{R}^{d}\right)$ the vector space $\mathbf{C}\left[x_{1}, \ldots, x_{d}\right]$ of polynomials on $\mathbf{R}^{d}$ with complex coefficients is contained in $L^{p}(\mu)$ for every $p \in\left[1, \infty\right.$ [. We denote the norm in $L^{p}(\mu)$ by $\|\cdot\|_{\mu, p}$.

For an indeterminate $\mu \in \mathcal{M}^{*}\left(\mathbf{R}^{d}\right)$ the set $[\mu]$ can be described via spectral measures of certain commuting families of self-adjoint operators, see Fuglede [7] for details. In dimension $d=1$ a much more precise result is available: The Nevanlinna parametrization, see Akhiezer [1] or Buchwalter and Cassier [5] for details. To describe it let $N$ be the set of Nevanlinna-Pick functions $\varphi: \mathbf{C} \backslash \mathbf{R} \rightarrow \mathbf{C}$ given by

$$
\varphi(z)=\alpha z+\beta+\int \frac{t z+1}{t-z} d \sigma(t)
$$

where $\alpha \geqslant 0, \beta \in \mathbf{R}$ and $\sigma$ is a positive finite measure on $\mathbf{R}$. Then $N \cup\{\infty\}$ can be used as parameter space for $[\mu]$ independent of the particular indeterminate $\mu \in \mathcal{M}^{*}(\mathbf{R})$ via the bijection $\varphi \mapsto \mu_{\varphi}$ of $N \cup\{\infty\}$ onto $[\mu]$ given by

$$
\int \frac{d \mu_{\varphi}(t)}{z-t}=\frac{A(z) \varphi(z)-C(z)}{B(z) \varphi(z)-D(z)}, \quad z \in \mathbf{C} \backslash \mathbf{R},
$$

where $A, B, C, D$ are certain entire functions associated with $[\mu]$.

The Nevanlinna extremal solutions correspond to the constant functions $\varphi(z) \equiv \beta$, with $\beta \in \mathbf{R} \cup\{\infty\}$. The Nevanlinna extremal solution $\mu_{0}$ corresponding to the parameter value $\beta=0$ plays an important role later. It is the only Nevanlinna extremal measure having mass at 0 , and it is given by

$$
\mu_{0}=\sum_{d \in \Lambda} \varrho(d) \varepsilon_{d}
$$

where $\Lambda$ is the discrete set of zeros of the entire function $D$ from the Nevanlinna parametrization. According to [1] 


$$
D(z)=z \sum_{k=0}^{\infty} P_{k}(0) P_{k}(z)
$$

and

$$
\varrho(z)=\left(\sum_{k=0}^{\infty}\left|P_{k}(z)\right|^{2}\right)^{-1}
$$

where $\left(P_{k}\right)_{k \geqslant 0}$ denote the orthonormal polynomials associated with $[\mu]$, uniquely determined by orthonormality and the requirement that the leading coefficient of each $P_{k}$ is positive.

In the Stieltjes case, where at least one of the measures $\sigma \in[\mu]$ is concentrated on [0, [, all the zeros of $P_{k}$ are positive so we have $(-1)^{k} P_{k}(x)>0$ for $x \leqslant 0$, hence $D(x)<0$ for $x<0$. The zero set $\Lambda$ for $D$ therefore consists of a sequence

$$
0=d_{0}<d_{1}<d_{2}<\ldots \rightarrow \infty
$$

so $\mu_{0}$ is supported by $[0, \infty[$. This yields a simple proof of the following known result:

Proposition 1.1. Let $\sigma \in \mathcal{M}^{*}([0, \infty[)$ be $\operatorname{det}(S)$ and indeterminate. Then $\sigma$ is equal to the Nevanlinna extremal solution $\sigma_{0}$.

Corollary 1.2. Let $\sigma \in M^{*}([0, \infty[)$ be $\operatorname{det}(S)$. Then the polynomials are dense in $L^{2}(\sigma)$

Proof. This is a consequence of the theorem of Riesz [14], because $\sigma$ is either determinate or the Nevanlinna extremal solution $\sigma_{0}$.

Corollary 1.2. is a preliminary result. For a stronger statement see Corollary 3.9.

We recall two classical results which will be used several times in the sequel:

Proposition 1.3 (Riesz [14]). Let $\mu \in \mathcal{M}^{*}(\mathbf{R})$. Then $\mu$ is determinate if and only if the polynomials are dense in $L^{2}\left(\left(1+x^{2}\right) d \mu(x)\right)$.

For a proof see also [7, p. 58].

In Theorem 3.8 below we characterize determinacy in the sense of Stieltjes by $L^{2}$-denseness in analogy with Proposition 1.3.

Proposition 1.4. Let $\mu \in \mathcal{M}^{*}(\mathbf{R})$ be indeterminate and Nevanlinna extremal and let $a \in \mathbf{R}$. If $\mu(\{a\})>0$ then $\nu=\mu-\mu(\{a\}) \varepsilon_{a}$ is determinate.

For a proof see $[1$, p. 115] or $[2$, p. 111]. 
Corollary 1.5. Let $\mu \in \mathcal{M}^{*}(\mathbf{R})$ be of the form $\mu=\alpha \varepsilon_{a}+v$ where $\alpha>0$ and $\nu(\{a\})=0$. Then $v$ is determinate if and only if the polynomials are dense in $L^{2}(\mu)$.

Proof. Assume first that $\mathbf{C}[x]$ is dense in $L^{2}(\mu)$. If $\mu$ is determinate, so is the smaller measure $v$. If $\mu$ is indeterminate it is Nevanlinna extremal, so that $v$ is determinate by Proposition 1.4.

Assume next that $v$ is determinate. Then $\mathbf{C}[x]$ is dense in $L^{2}(\mu)$ by a result of Berg and Christensen, cf. [2, p. 113].

\section{Rotation invariant measures and their moments}

The orthogonal group $O(d)$ operates continuously in the convex cone $M\left(\mathbf{R}^{d}\right)$ of positive Radon measures on $\mathbf{R}^{d}$ in the following way: If $\mu \in M\left(\mathbf{R}^{d}\right)$ and $R \in O(d)$ then $\mu^{R} \in M\left(\mathbf{R}^{d}\right)$ is the image measure of $\mu$ under $R$.

We say that $\mu \in M\left(\mathbf{R}^{d}\right)$ is rotation invariant if $\mu^{R}=\mu$ for all $R \in O(d)$ and denote by $M_{\text {rot }}\left(\mathbf{R}^{d}\right)$ the set of rotation invariant positive Radon measures on $\mathbf{R}^{d}$.

Let $d R$ refer to the normalized Haar measure on $O(d)$, and let $\Omega_{d}$ denote the unit sphere in $\mathbf{R}^{d}$. For $\xi \in \Omega_{d}$ and $\varphi \in C\left(\Omega_{d}\right)$ we have

$$
\int_{O(d)} \varphi(R \xi) d R=\int_{\Omega_{d}} \varphi d \omega
$$

where $\omega$ is the normalized surface measure of $\Omega_{d}$ characterized by being the only rotation invariant probability on $\Omega_{d}$.

For $\mu \in M\left(\mathbf{R}^{d}\right)$ the vector integral

$$
\tilde{\mu}=\int_{O(d)} \mu^{R} d R
$$

defines a measure $\tilde{\mu} \in M_{\text {rot }}\left(\mathbf{R}^{d}\right)$ such that

$$
\int f d \tilde{\mu}=\int_{O(d)}\left(\int_{\mathbf{R}^{d}} f(R x) d \mu(x)\right) d R, f \in C_{\mathfrak{c}}\left(\mathbf{R}^{d}\right) .
$$

Let $\psi: \mathbf{R}^{d} \rightarrow \mathbf{R}$ and $j:\left[0, \infty\left[\times \Omega_{d} \rightarrow \mathbf{R}^{d}\right.\right.$ denote the functions

$$
\psi(x)=\|x\|^{2}=\sum_{i=1}^{d} x_{i}^{2}, \quad j(t, \xi)=\sqrt{t} \xi
$$

Using (2.1) we find 


$$
\begin{aligned}
\int f d \tilde{\mu} & =\int_{\Omega_{d}} \int_{\mathbf{R}^{d}} f(\|x\| \xi) d \mu(x) d \omega(\xi) \\
& =\int_{\Omega_{d}} \int_{0}^{\infty} f(\sqrt{t} \xi) d \mu^{\psi}(t) d \omega(\xi)=\int f d\left(\mu^{\psi} \otimes \omega\right)^{j}
\end{aligned}
$$

showing that

$$
\hat{\mu}=\left(\mu^{\psi} \otimes \omega\right)^{j} \text { for } \mu \in M\left(\mathbf{R}^{\Phi}\right),
$$

i.e. $\bar{\mu}$ is the image measure under $j$ of the product of the measures $\mu^{\psi}$ and $\omega$. In particular we have

$$
\mu=\left(\mu^{\psi} \otimes \omega\right)^{j} \quad \text { for } \quad \mu \in M_{\text {rot }}\left(\mathbf{R}^{d}\right)
$$

For $\sigma \in M\left(\left[0, \infty[)\right.\right.$ the measure $\mu=(\sigma \otimes \omega)^{j}$ belongs to $M_{\mathrm{rot}}\left(\mathbf{R}^{d}\right)$ and $\mu^{\psi}=\sigma$ as is easily seen.

We may summarize the above formulas in the following way:

Proposition 2.1. Let $\psi$ and $j$ be defined by (2.3). Then $\mu \mapsto \mu^{\psi}$ is a bijection of $M_{\text {rot }}\left(\mathbf{R}^{d}\right)$ onto $M\left(\left[0, \infty[)\right.\right.$, and the inverse mapping is $\sigma \mapsto(\sigma \otimes \omega)^{j}$.

Let $\mu \in M_{\text {rot }}\left(\mathbf{R}^{d}\right)$. If $\mu \in \mathscr{M}^{*}\left(\mathbf{R}^{d}\right)$ then $\mu^{\psi} \in \mathcal{M}^{*}([0, \infty[)$ and

$$
\int t^{n} d \mu^{\psi}(t)=\int\|x\|^{2 n} d \mu(x)
$$

Conversely, if $\sigma \in \mathcal{M}^{*}([0, \infty])$ then $\mu=(\sigma \otimes \omega)^{j} \in \mathcal{M}^{*}\left(\mathbf{R}^{d}\right)$ and

$$
\int x^{\alpha} d \mu(x)=\int_{0}^{\infty} t^{\mid \alpha / 2} d \sigma(t) \int_{\Omega_{d}} \xi^{\alpha} d \omega(\xi)
$$

It is clear that $\int \xi^{\alpha} d \omega(\xi)=0$ if at least one $\alpha_{i}$ is odd, and it is easy to see that

$$
\int_{\Omega_{d}} \xi^{2 \alpha} d \omega(\xi)=\frac{\Gamma\left(\alpha_{1}+1 / 2\right) \ldots \Gamma\left(\alpha_{d}+1 / 2\right)}{\Gamma\left(\alpha_{1}+\ldots+\alpha_{d}+d / 2\right)} \Gamma\left(\frac{d}{2}\right) \pi^{-d / 2}
$$

This shows that $\mathcal{M}_{\mathrm{rot}}^{*}\left(\mathbf{R}^{d}\right):=\mathcal{M}^{*}\left(\mathbf{R}^{d}\right) \cap M_{\mathrm{rot}}\left(\mathbf{R}^{d}\right)$ is in one-to-one correspondence with $\mathscr{M}^{*}([0, \infty[)$ under the bijection of Proposition 2.1 , and that the non-trivial moments $s(\alpha)=\int x^{\alpha} d \mu(x)$ (all $\alpha_{i}$ even) of $\mu \in \mathcal{M}_{\mathrm{rot}}^{*}\left(\mathbf{R}^{d}\right)$ are expressed as in (2.7) in terms of the Stieltjes moments of the corresponding measure $\sigma=\mu^{\psi}$ on $[0, \infty[$.

The $d$-dimensional moment problem consists in characterizing those sequences $s: \mathbf{N}^{d} \rightarrow \mathbf{R}$ which are the moment sequence of some measure $\mu \in \mathcal{M}^{*}\left(\mathbf{R}^{d}\right)$. 
To $s: \mathbf{N}^{d} \rightarrow \mathbf{R}$ we associate the $\mathbf{C}$-linear functional $L_{s}: \mathbf{C}\left[x_{1}, \ldots, x_{d}\right] \rightarrow \mathbf{C}$ on the space of complex polynomials given by

$$
L_{s}\left(x^{\alpha}\right)=s(\alpha), \quad \alpha \in \mathbf{N}^{d} .
$$

We recall the following results (see e.g. [3]):

(i) $s$ is a moment sequence if and only if $L_{s}$ is non-negative on non-negative polynomials.

(ii) If $s$ is a moment sequence then $s$ is positive definite on the semigroup $\mathbf{N}^{d}$, (i.e. the kernel $(\alpha, \beta) \mapsto s(\alpha+\beta)$ is positive semidefinite on $\left.\mathbf{N}^{d} \times \mathbf{N}^{d}\right)$.

(iii) Let $s$ be positive definite on $\mathrm{N}^{d}$. Then $s$ is a moment sequence if $d=1$ (Hamburger's theorem), but $s$ need not be a moment sequence if $d>1$.

We shall see that in the rotation invariant case positive definiteness of $s$ is sufficient for $s$ to be a moment sequence.

Proposition 2.2. The following properties of a function $s: \mathbf{N}^{d} \rightarrow \mathbf{R}$ are equivalent:

(i) $s$ is the moment sequence of a measure $\mu \in \mathcal{M}_{\mathrm{rot}}^{*}\left(\mathbf{R}^{d}\right)$.

(ii) $s$ is positive definite and $L_{s}$ is rotation invariant in the sense that $L_{s}(p \circ R)=L_{s}(p)$ for $p \in \mathbf{C}\left[x_{1}, \ldots, x_{d}\right]$ and $R \in O(d)$.

(iii) There exists a Stieltjes moment sequence $t$ such that

$$
s(\alpha)= \begin{cases}0, & \alpha_{i} \text { is odd for some } i=1, \ldots, d, \\ t\left(\frac{1}{2}|\alpha|\right) \int_{\Omega_{d}} \xi^{\alpha} d \omega(\xi), & \alpha_{i} \text { is even for every } i=1, \ldots, d .\end{cases}
$$

Proof. The implication "(i) $\Rightarrow$ (ii)" is obvious.

To prove that "(ii) $\Rightarrow$ (iii)" we define $t(n)=L_{s}\left(\|x\|^{2 n}\right), n \geqslant 0$, and want to show that $t$ is a Stieltjes moment sequence. We shall show that $t$ and the shifted sequence $(E t)(n)=t(n+1)$ are both positive definite. For $c_{0}, c_{1}, \ldots, c_{n} \in \mathbf{C}$ we have

$$
\sum_{i, j=0}^{n} t(i+j) c_{j} \bar{c}_{j}=L_{s}\left(\left|\sum_{i=0}^{n} c_{i}\|x\|^{2 i}\right|^{2}\right) \geqslant 0
$$

and

$$
\sum_{i, j=0}^{n} t(i+j+1) c_{i} \bar{c}_{j}=\sum_{k=1}^{d} L_{s}\left(\left|\left(\sum_{i=0}^{n} c_{i}\|x\|^{2 i}\right) x_{k}\right|^{2}\right) \geqslant 0
$$

where we have used that $L_{s}\left(|p|^{2}\right) \geqslant 0$ for any $p \in \mathbf{C}\left[x_{1}, \ldots, x_{d}\right]$, since $s$ is positive defhite. 
We next have to show that the equality in (iii) holds. Let $\alpha \in \mathbf{N}^{d}$. If some of the coordinates of $\alpha$ are odd we find

$$
s(\alpha)=L_{s}\left(x^{\alpha}\right)=0=\int_{\Omega_{d}} \xi^{\alpha} d \omega(\xi),
$$

by considering the reflection in a coordinate in which $\alpha$ is odd and using the rotation invariance. We can thus restrict our attention to the case where $\alpha$ is of the form $\alpha=2 \beta$ with $\beta \in \mathbf{N}^{d}$. We have to show that

$$
L_{s}\left(x^{2 \beta}\right)=L_{s}\left(\|x\|^{2|\beta|}\right) \int_{\Omega_{d}} \xi^{2 \beta} d \omega(\xi) .
$$

For any polynomial $p \in \mathbf{C}\left[x_{1}, \ldots, x_{d}\right]$ we define a rotation invariant polynomial $\tilde{p}$ by the vector integral

$$
\tilde{p}=\int_{O(d)} p \circ R d R
$$

and since $L_{s}$ is rotation invariant we have $L_{s}(\tilde{p})=L_{s}(p)$. For $p(x)=x^{2 \beta}$ we find

$$
\tilde{p}(x)=\int_{O(d)} p(R x) d R=\int_{\Omega_{d}} p(\|x\| \xi) d \omega(\xi)=\|\left. x\right|^{|2 \beta|} \int_{\Omega_{d}} \xi^{2 \beta} d \omega(\xi),
$$

and (2.8) follows.

Assume finally that (iii) holds and let $\sigma \in \mathcal{M}^{*}([0, \infty[)$ be a representing measure for $t$. Then $\mu=(\sigma \otimes \omega)^{j}$ belongs to $\mathcal{M}_{\mathrm{rot}}^{*}\left(\mathbf{R}^{d}\right)$, and its moment sequence is easily seen to be $s$.

We next want to show how to obtain orthogonal polynomials with respect to a rotation invariant measure. This constitutes a natural extension of the results in $[6$, section I].

Let $\mu \in M_{\mathrm{rot}}^{*}\left(\mathbf{R}^{d}\right), d>1$, and let $\sigma=\mu^{\psi}$. For $k \in \mathbf{N}$ let $\left(P_{k, n}(t)\right)_{n \in \mathrm{N}}$ denote the system of orthonormal polynomials associated with the measure $t^{k} d \sigma(t)$. Let $\mathscr{H}_{d, k}$ denote the space of spherical harmonics (i.e. homogeneous harmonic polynomials) of degree $k$ on $\mathbf{R}^{d}$. Let $S_{k, j}, j=1, \ldots, \operatorname{dim} \mathscr{H}_{d, k}$ denote an orthonormal basis of $\mathscr{H}_{d, k}$ considered as a subspace of $L^{2}(\omega)$.

For $k, n \in \mathrm{N}$ and $j=1, \ldots, \operatorname{dim} \mathscr{H}_{d, k}$ we define

$$
p_{k, n, j}(x)=\boldsymbol{P}_{k, n}\left(\|x\|^{2}\right) S_{k, j}(x) \in \mathbf{C}\left[x_{1}, \ldots, x_{d}\right] .
$$


These polynomials form an orthonormal basis of $\mathbf{C}\left[x_{1}, \ldots, x_{d}\right]$ considered as a subspace of $L^{2}(\mu)$. Indeed every polynomial in $\mathbf{C}\left[x_{1}, \ldots, x_{d}\right]$ is a sum of polynomials of the form $p\left(\|x\|^{2}\right) S(x)$, where $p(t) \in \mathrm{C}[t]$ and $S(x)$ is a spherical harmonic, cf. [16, p. 139]. Moreover one has

$$
\int_{\mathbf{R}^{d}} p_{k, n, j} \overline{p_{k^{\prime}, n^{\prime}, j^{\prime}}} d \mu=\int_{0}^{\infty} P_{k, n}(t) P_{k^{\prime}, n^{\prime}}(t) t^{\left(k+k^{\prime}\right) / 2} d \sigma(t) \int_{\Omega_{d}} S_{k, j} \overline{S_{k^{\prime}, j^{\prime}}} d \omega .
$$

The last factor vanishes when $(k, j) \neq\left(k^{\prime}, j^{\prime}\right)$. If $k=k^{\prime}$ and $j=j^{\prime}$ the above expression equals

$$
\int_{0}^{\infty} P_{k, n}(t) P_{k, n^{\prime}}(t) t^{k} d \sigma(t)=\delta_{n n^{\prime}}
$$

\section{Main results}

We first give a set of functions which is total in $L^{p}(\mu)$, when $p \in[1, \infty[$ and $\mu$ is an arbitrary measure in $M\left(\mathbf{R}^{d}\right)$.

Proposition 3.1. Let $\mu \in M\left(\mathbf{R}^{d}\right)$ and $p \in[1, \infty[$. The linear span of the functions $f\left(\|x\|^{2}\right) S(x)$, where $f \in C_{\mathrm{c}}([0, \infty[)$ and $S(x)$ is a spherical harmonic, is a dense subspace in $L^{p}(\mu)$.

Proof. By disintegration of $\mu$ with respect to the mapping $\psi: x \mapsto\|x\|^{2}$ from $\mathbf{R}^{d}$ onto $[0, \infty[$ we get

$$
\mu=\int_{0}^{\infty} \lambda_{t} d \sigma(t)
$$

where $\sigma=\mu^{\psi}$, and $\lambda_{1}$ is a probability measure on the sphere

$$
\left\{x \in \mathbf{R}^{d} \mid\|x\|=\sqrt{t}\right\}
$$

for $t \in\left[0, \infty\left[\right.\right.$, cf. Bourbaki [4, Chapter 6, p. 58]. Assume that $g \in L^{q}(\mu)$ annihilates all the functions in question, $q$ being the dual exponent of $p$. We shall show that $g=0 \mu$-a.e.. By assumption we have

$$
\begin{aligned}
0 & =\int f\left(\|x\|^{2}\right) S(x) g(x) d \mu(x) \\
& =\int_{0}^{\infty}\left(f(t) \int S g d \lambda_{t}\right) d \sigma(t),
\end{aligned}
$$


and since $f \in C_{\mathrm{c}}([0, \infty[)$ is arbitrary, we get for every spherical harmonic $S$ that

$$
\int_{\|x\|=\sqrt{t}} S g d \lambda_{t}=0
$$

for $\sigma$-almost all $t \in\left[0, \infty\right.$ [. Using a (countable) orthonormal basis for $L^{2}(\omega)$ of spherical harmonics we see that there exists a $\sigma$-null set $N \subset[0, \infty[$ such that (3.1) holds for all spherical harmonics $S$ and for all $t \in[0, \infty[\backslash N$. The linear span of the set of restrictions of spherical harmonics $S$ to the sphere $\|x\|=\sqrt{t}$ is uniformly dense in the continuous functions on the sphere, cf. [16, p. 139], and we therefore get $g=0 \lambda_{t}$-a.e. for $\sigma$-almost all $t$, hence $g=0 \mu$-a.e.

THEOREM 3.2. Let $\mu \in \mathcal{M}^{*}\left(\mathbf{R}^{d}\right)$ where $d>1$, define $\sigma=\mu^{\psi}$ and let $p \in[1, \infty[$. If $\mathbf{C}[t]$ is dense in $L^{p}\left(t^{k p / 2} d \sigma(t)\right)$ for every integer $k \geqslant 0$ then $\mathbf{C}\left[x_{1}, \ldots, x_{d}\right]$ is dense in $L^{p}(\mu)$.

The converse holds when $\mu$ is rotation invariant and $p=2$.

Proof. Assume first that $\mathbf{C}[t]$ is dense in $L^{p}\left(t^{k p / 2} d \sigma(t)\right)$ for every integer $k \geqslant 0$. To see that $\mathbf{C}\left[x_{1}, \ldots, x_{d}\right]$ is dense in $L^{p}(\mu)$, it suffices by Proposition 3.1 to approximate $f\left(\|x\|^{2}\right) S(x)$ arbitrarily well in $L^{p}(\mu)$ by polynomials, $f \in C_{\mathrm{c}}([0, \infty[)$ and $S(x)$ a spherical harmonic being given. Let $k$ be the degree of $S(x)$. For $h(t) \in \mathbf{C}[t]$ we define $Q(x)=h\left(\|x\|^{2}\right) S(x) \in \mathbf{C}\left[x_{1}, \ldots, x_{d}\right]$ and notice that

$$
\begin{aligned}
\left\|f\left(\|x\|^{2}\right) S(x)-Q(x)\right\|_{\mu, p} & =\left\|\left(f\left(\|x\|^{2}\right)-h\left(\|x\|^{2}\right)\right)\right\| x\left\|^{k} S(x /\|x\|)\right\|_{\mu, p} \\
& \leqslant \sup _{\xi \in \Omega_{d}}|S(\xi)| \cdot\left\|\left(f\left(\|x\|^{2}\right)-h\left(\|x\|^{2}\right)\right)\left(\|x\|^{2}\right)^{k / 2}\right\|_{\mu, p},
\end{aligned}
$$

which can be made as small as we please since the second factor equals $\|f-h\|_{\tau, p}$, where for simplicity of notation we have put

$$
\tau=t^{k p / 2} d \sigma(t)
$$

and we assume that $\mathbf{C}[t]$ is dense in $L^{p}(\tau)$.

Assume next that $\mu$ is rotation invariant, $p=2$ and that $\mathbf{C}[t]$ is not dense in $L^{2}\left(t^{k} d \sigma(t)\right)$ for some integer $k \geqslant 0$. Then there is a function $f \in L^{2}\left(t^{k} d \sigma(t)\right) \backslash\{0\}$ which is orthogonal to $\mathbf{C}[t]$. Let $S_{k}(x)$ be a non-zero spherical harmonic of degree $k$ on $\mathbf{R}^{d}$. Then $f\left(\|x\|^{2}\right) S_{k}(x)$ belongs to $L^{2}(\mu) \backslash\{0\}$, because by (2.5) we have

$$
\left\|f\left(\|x\|^{2}\right) S_{k}(x)\right\|_{\mu, 2}=\|f\|_{t^{k} d o(t), 2}\left\|S_{k}\right\|_{\omega, 2} .
$$

We want to show that $f\left(\|x\|^{2}\right) S_{k}(x)$ is orthogonal to $\mathrm{C}\left[x_{1}, \ldots, x_{d}\right]$. Every polynomial in 15-918289 Acta Mathematica 167. Imprimé le 5 novembre 1991 
$\mathbf{C}\left[x_{1}, \ldots, x_{d}\right]$ is a sum of polynomials of the form $p\left(\|x\|^{2}\right) S(x)$ where $p(t) \in \mathbf{C}[t]$ and $S(x)$ is a spherical harmonic, cf. [16, p. 139]. If the degree of $S(x)$ is $m$, we have (again by (2.5))

$$
\int f\left(\|x\|^{2}\right) S_{k}(x) \overline{p\left(\|x\|^{2}\right) S(x)} d \mu(x)=\int_{0}^{\infty} f(t) \overline{p(t)} t^{(k+m) / 2} d \sigma(t) \int_{\Omega_{d}} S_{k}(\xi) \overline{S(\xi)} d \omega(\xi) .
$$

Here, the second factor vanishes when $m \neq k$, and the first factor vanishes when $m=k$.

On the real line there exist spherical harmonics of degree 0 and 1 only. For $d=1$ one obtains therefore the following result from Proposition 3.1:

Theorem 3.3. Let $\mu \in \mathcal{M}^{*}(\mathbf{R})$, define $\sigma=\mu^{\psi}$ and let $p \in[1, \infty[$. If $\mathbf{C}[t]$ is dense in $L^{p}(\sigma)$ and in $L^{p}\left(t^{p / 2} d \sigma(t)\right)$ then $\mathrm{C}[x]$ is dense in $L^{p}(\mu)$.

The converse holds when $\mu$ is symmetric and $p=2$.

Corollary 3.4. Let $\mu \in \mathcal{M}^{*}\left(\mathbf{R}^{d}\right)$ and consider the measure $\tilde{\mu}$ defined by (2.2). If $\mathbf{C}\left[x_{1}, \ldots, x_{d}\right]$ is dense in $L^{2}(\tilde{\mu})$, then $\mathbf{C}\left[x_{1}, \ldots, x_{d}\right]$ is dense in $L^{2}(\mu)$.

This result is obvious for $d=1$ because in that case $\mu \leqslant 2 \bar{\mu}$.

To establish (1) from the introduction we need

Proposition 3.5. Let $\mu \in \mathcal{M}^{*}\left(\mathbf{R}^{d}\right)$ and put $\sigma=\mu^{\psi}$. Assume that $\mathbf{C}[t]$ is dense in $L^{p}(\sigma)$ for some $p \in] 1, \infty\left[\right.$. Then $\mathbf{C}\left[x_{1}, \ldots, x_{d}\right]$ is dense in $L^{p^{\prime}}(\mu)$ for every $p^{\prime} \in[1, p[$.

Proof. Let $p^{\prime} \in[1, p[$. By Theorems 3.2. and 3.3 it suffices to show that $\mathbf{C}[t]$ is dense in $L^{p^{\prime}}\left(t^{k p^{\prime} / 2} d \sigma(t)\right)$ for every integer $k \geqslant 0$. Let $k \in \mathbf{N}$ and $f \in C_{\mathrm{c}}([0, \infty[)$. For $h(t) \in \mathbf{C}[t]$ we have

$$
\int|f(t)-h(t)|^{p^{\prime}} t^{k^{\prime} / 2} d \sigma(t) \leqslant\left(\int|f(t)-h(t)|^{p} d \sigma(t)\right)^{p^{\prime \prime p}}\left(\int t^{k\left(p^{\prime} / 2\right) s} d \sigma(t)\right)^{1 / s},
$$

where $s$ is the dual exponent of $p / p^{\prime}$ in Hölder's inequality. The first factor can be made arbitrarily small, because we assume that $\mathbf{C}[t]$ is dense in $L^{p}(\sigma)$.

The following theorem (which establishes (1)) is well-known in the one-dimensional symmetric case, see e.g. Heyde [9, p. 92] or Chihara [6, p. 333]. Their proofs are based upon the Nevanlinna parametrization and are not applicable in the multidimensional case. Our proof parallels Petersen's proof that $\mu \in \mathcal{M}^{*}\left(\mathbf{R}^{d}\right)$ is determinate if its marginal distributions are determinate, cf. [13, Theorem 3]. 
TheORem 3.6. Let $\mu \in \mathcal{M}^{*}\left(\mathbf{R}^{d}\right)$ and put $\sigma=\mu^{\psi}$. If $\sigma$ is $\operatorname{det}(S)$ then $\mu$ is determinate.

The converse holds if $\mu \in \mathcal{M}_{\mathrm{rot}}^{*}\left(\mathbf{R}^{d}\right)$.

Proof. We shall need that a measure $v \in[\mu]$ is an extreme point of $[\mu]$ if and only if the polynomials are dense in $L^{1}(v)$. This is shown in [1, p. 47] for $d=1$, but the proof applies in any dimension.

Assume that $\sigma$ is $\operatorname{det}(\mathrm{S})$. For $\nu \in[\mu]$ we have $v^{\psi} \sim \sigma$ by (2.6), hence $\nu^{\psi}=\sigma$. Since the polynomials are dense in $L^{2}(\sigma)=L^{2}\left(\nu^{\psi}\right)$ by Corollary 1.2, it follows by Proposition 3.5 that $\mathbf{C}\left[x_{1}, \ldots, x_{d}\right]$ is dense in $L^{1}(v)$, so that $\nu$ is an extreme point of $[\mu]$. The measure $v \in[\mu]$ being arbitrary, this is only possible if $\mu$ is determinate.

Assume next that $\mu \in \mathcal{M}_{\mathrm{rot}}^{*}\left(\mathbf{R}^{d}\right)$ and that $\sigma$ is indet(S). Then $\mu$ is indeterminate, because if $\tau \in \mathscr{M}^{*}\left(\left[0, \infty[)\right.\right.$ is equivalent to $\sigma$ then $(\tau \otimes \omega)^{j} \sim \mu$, by (2.7).

Corollary 3.7. Let $\mu \in \mu^{*}\left(\mathbf{R}^{d}\right)$ and consider the measure $\tilde{\mu}$ defined by (2.2). If $\tilde{\mu}$ is determinate then $\mu$ is determinate.

This result is obvious for $d=1$ because in that case $\mu \leqslant 2 \tilde{\mu}$.

We shall now give the analogue of Proposition 1.3 for the Stieltjes case.

TheOREM 3.8. Let $\sigma \in \mathscr{M}^{*}([0, \infty[)$. Then $\sigma$ is $\operatorname{det}(S)$ if and only if $\mathbf{C}[t]$ is dense in $L^{2}((1+t) d \sigma(t))$ and in $L^{2}(t(1+t) d \sigma(t))$.

Proof. Let $\mu \in \mathcal{M}^{*}(\mathbf{R})$ be the unique symmetric measure for which $\mu^{\psi}=\sigma$. By Theorem 3.6 the measure $\sigma$ is $\operatorname{det}(\mathbf{S})$ precisely when $\mu$ is determinate. By Proposition 1.3 the measure $\mu$ is determinate if and only if $\mathbf{C}[x]$ is dense in $L^{2}\left(\left(1+x^{2}\right) d \mu(x)\right)$, and by Theorem 3.3 this is equivalent to $\mathbf{C}[t]$ being dense in $L^{2}((1+t) d \sigma(t))$ and in $L^{2}(t(1+t) d \sigma(t))$.

Corollary 3.9. If $\sigma \in \mathcal{M}^{*}\left(\left[0, \infty[)\right.\right.$ is $\operatorname{det}(S)$, then $\mathrm{C}[t]$ is dense in $L^{2}(\sigma), L^{2}(t \operatorname{do}(t))$ and in $L^{2}\left(t^{2} d \sigma(t)\right)$.

Corollary 3.10. Let $\mu \in \mathcal{M}_{\mathrm{rot}}^{*}\left(\mathbf{R}^{d}\right)$ be determinate. Then the polynomials are dense in $L^{p}\left(\left(1+\|x\|^{2}\right) d \mu(x)\right)$ and in $L^{p}\left(\|x\|^{2}\left(1+\|x\|^{2}\right) d \mu(x)\right)$ for any $p \in[1,2[$. In particular the polynomials are dense in $L^{p}(\mu)$ for any such $p$.

Proof. This follows immediately by combination of Theorem 3.6, Theorem 3.8 and Proposition 3.5.

Proposition 3.11. Let $\mu \in \mathcal{M}^{*}\left(\mathbf{R}^{d}\right)$ have a density with respect to Lebesgue measure. If $\mathbf{C}[t]$ is dense in $L^{2}\left(\mu^{\psi}\right)$ then $\mathbf{C}\left[x_{1}, \ldots, x_{d}\right]$ is dense in $L^{2}(\mu)$. 
Proof. The measure $\sigma=\mu^{\psi}$ is not discrete, because spheres are Lebesgue null sets. By Theorems 3.2 and 3.3 it suffices to prove that $\mathbf{C}[t]$ is dense in $L^{2}\left(t^{k} d \sigma(t)\right)$ for any integer $k \geqslant 0$. Assume the contrary. Then there is a smallest $k \in \mathbf{N}$ such that $\mathbf{C}[t]$ is not dense in $L^{2}\left(t^{k} d \sigma(t)\right)$. By assumption $k \geqslant 1$. The measure $\tau=t^{k-1} d \sigma(t)$ is indet(S) and Nevanlinna extremal, but then $\tau$ and $\sigma$ are discrete, which is a contradiction.

\section{Solution of the Challifour-Devinatz problem}

It follows from Theorems 3.2 and 3.6 that $\mu \in \mathcal{M}_{\mathrm{rot}}^{*}\left(\mathbf{R}^{d}\right), d>1$, is determinate and the polynomials are not dense in $L^{2}(\mu)$ if and only if $\mu=(\sigma \otimes \omega)^{j}$, where $\sigma \in \mathcal{M}^{*}([0, \infty[)$ is $\operatorname{det}(\mathbf{S})$ such that the polynomials are not dense in $L^{2}\left(t^{k} d \sigma(t)\right)$ for some $k \in \mathbf{N}$ (necessarily $\geqslant 3$ by Corollary 3.9).

We show below that such measures $\sigma$ exist, and we characterize them completely in the next section. We need the following result:

Proposition 4.1. Let $\sigma \in \mathcal{M}^{*}([0, \infty[)$ be indet $(S)$. Then $\mathbf{C}[t]$ is not dense in $L^{2}\left(t^{k} d \sigma(t)\right)$ for any integer $k \geqslant 3$.

Proof. Suppose that $\mathbf{C}[t]$ is dense in $L^{2}\left(t^{k} d \sigma(t)\right)$ for some integer $k \geqslant 3$. The measure $v=t^{k} d \sigma(t)$ is then indeterminate and Nevanlinna extremal, hence discrete. It is clear that $0 \notin \operatorname{supp}(v)$. Since $\left(1+t^{2}\right)^{-1} t^{2}$ increases with $t^{2}$, we have

$$
\frac{a^{2}}{1+a^{2}} \frac{1}{t^{2}} \leqslant \frac{1}{1+t^{2}} \text { for } t \in \operatorname{supp}(v)
$$

where $a>0$ is the smallest element of $\operatorname{supp}(v)$. We then have

$$
\frac{a^{2}}{1+a^{2}} t^{k-2} d \sigma(t)=\frac{a^{2}}{1+a^{2}} \frac{1}{t^{2}} d v(t) \leqslant \frac{1}{1+t^{2}} d v(t) .
$$

Since $v$ is Nevanlinna extremal, we know by Proposition 1.3 that the measure $\left(1+t^{2}\right)^{-1} d v(t)$ is determinate. By the inequality above $t^{k-2} d \sigma(t)$ is also determinate, which is impossible because $\sigma$ and hence $t^{k-2} d \sigma(t)$ is indet(S).

From Theorems 3.2 and 3.6 we immediately get the following consequence of Proposition 4.1:

CoRollary 4.2. If $\mu \in \mathcal{M}_{\mathrm{rot}}^{*}\left(\mathbf{R}^{d}\right), d>1$, is indeterminate, then the polynomials are not dense in $L^{2}(\mu)$.

This is in contrast with the one-dimensional case, where an indeterminate symmet- 
ric moment problem always has (precisely two) symmetric Nevanlinna extremal solutions, cf. [6].

We next show that the closure of $\mathbf{C}\left[x_{1}, \ldots, x_{d}\right]$ in $L^{2}(\mu)$ is "small" in $L^{2}(\mu)$, if it is not equal to $L^{2}(\mu)$.

THEOREM 4.3. Let $\mu \in \mathcal{M}_{\mathrm{rot}}^{*}\left(\mathbf{R}^{d}\right), d>1$. If the polynomials are not dense in $L^{2}(\mu)$, then the closure of $\mathbf{C}\left[x_{1}, \ldots, x_{d}\right]$ has infinite co-dimension in $L^{2}(\mu)$.

Proof. Assume that the polynomials are not dense in $L^{2}(\mu)$ and let $\sigma=\mu^{\psi}$. Then $\mathbf{C}[t]$ is not dense in $L^{2}\left(t^{n} d \sigma(t)\right)$ for some $n \in \mathrm{N}$, cf. Theorem 3.2. By Corollary 3.9 it follows that $t^{n} d \sigma(t)$ is indet(S), and Proposition 4.1 implies that $\mathbf{C}[t]$ is not dense in $L^{2}\left(t^{k} d \sigma(t)\right)$ for $k \in \mathbf{N}, k \geqslant n+3$. For each of these infinitely many values of $k$, let $f_{k} \in L^{2}\left(t^{k} d \sigma(t)\right) \backslash\{0\}$ be orthogonal to $\mathbf{C}[t]$ and let $S_{k}(x)$ be a non-zero spherical harmonic of degree $k$ on $\mathbf{R}^{d}$. The functions $f_{k}\left(\|x\|^{2}\right) S_{k}(x) \in L^{2}(\mu) \backslash\{0\}$ then belong to the orthogonal complement of $\mathrm{C}\left[x_{1}, \ldots, x_{d}\right]$ in $L^{2}(\mu)$, cf. the proof of Theorem 3.2. Moreover, these functions are pairwise orthogonal because

$$
\int f_{k}\left(\|x\|^{2}\right) S_{k}(x) \overline{f_{k^{\prime}}\left(\|x\|^{2}\right) S_{k^{\prime}}(x)} d \mu(x)=\int_{0}^{\infty} f_{k}(t) \overline{f_{k^{\prime}}(t)} t^{\left(k+k^{\prime}\right) / 2} d \sigma(t) \int_{\Omega_{d}} S_{k}(\xi) \overline{S_{k^{\prime}}(\xi)} d \omega(\xi),
$$

and the second factor vanishes when $k^{\prime} \neq k$.

If $\sigma \in \mathcal{M}^{*}\left(\left[0, \infty[)\right.\right.$ is indeterminate, then $\sigma_{0}$ also belongs to $\mathcal{M}^{*}([0, \infty[)$ and is given by

$$
\sigma_{0}=\sum_{n=0}^{\infty} \varrho\left(d_{n}\right) \varepsilon_{d_{n}}
$$

where $0=d_{0}<d_{1}<d_{2}<\ldots$ are the zeros of the function $D$, cf. (1.4).

The following class $\mathscr{S}$ of measures ( $\mathscr{S}$ for Stieltjes) will be crucial for the solution of the Challifour-Devinatz problem.

$$
\mathscr{S}=\left\{\sigma_{0}-\sigma_{0}(\{0\}) \varepsilon_{0} \mid \sigma \in \mathscr{M}^{*}([0, \infty[) \text { is } \operatorname{indet}(\mathrm{S})\} .\right.
$$

With $\sigma_{0}$ as above the measure $\nu=\sigma_{0}-\sigma_{0}(\{0\}) \varepsilon_{0} \in \mathscr{S}$ is of the form

$$
v=\sum_{n=1}^{\infty} \varrho\left(d_{n}\right) \varepsilon_{d_{n}}
$$

and it is determinate by Proposition 1.4. Since $t^{k} d v(t)=t^{k} d \sigma_{0}(t)$ for $k \geqslant 1$ and $\sigma_{0}$ is indet(S), Proposition 4.1 implies that $\mathrm{C}[t]$ is not dense in $L^{2}\left(t^{k} d v(t)\right)$ for any $k \geqslant 3$. The 
corresponding rotation invariant measure $\mu=(\nu \otimes \omega)^{j}$ has the form

$$
\mu=\sum_{n=1}^{\infty} \varrho\left(d_{n}\right) \omega_{\sqrt{d_{n}}},
$$

where $\omega_{r}$ is the normalized uniform distribution on the sphere $\|x\|=r$ in $\mathbf{R}^{d}$. We have thereby shown the existence part of the following solution in the negative of the Challifour-Devinatz problem from the introduction.

THEOREM 4.4. There exist determinate measures $\mu \in \mathcal{M}_{\mathrm{rot}}^{*}\left(\mathbf{R}^{d}\right), d>1$, for which $\mathbf{C}\left[x_{1}, \ldots, x_{d}\right]$ is not dense in $L^{2}(\mu)$. Such measures are necessarily of the form

$$
\mu=\sum_{n=1}^{\infty} \alpha_{n} \omega_{r_{n}}
$$

where $\alpha_{n}>0,0 \leqslant r_{0}<r_{1}<\ldots \rightarrow \infty$.

Proof. We have to prove that if $\mu \in \mathcal{M}_{\mathrm{rot}}^{*}\left(\mathbf{R}^{d}\right)$ is determinate and the polynomials are not dense in $L^{2}(\mu)$, then $\mu$ has the special form of the theorem. By Theorems 3.2. and 3.6 we see that $\sigma=\mu^{\psi}$ is $\operatorname{det}(S)$ and there exists an integer $k(\geqslant 3)$, such that $\mathbf{C}[t]$ is not dense in $L^{2}\left(t^{k} d \sigma(t)\right)$. Let $k_{0}$ be the smallest integer with this property. Then $\tau=t^{k_{0}-1} d \sigma(t)$ is such that $\mathbf{C}[t]$ is dense in $L^{2}(\tau)$ but not in $L^{2}(t d \tau(t))$, so $\tau$ is indet(S) by Corollary 3.9 and hence Nevanlinna extremal and in particular discrete. The mass points of $\sigma$ and $\tau$ are the same except that $\sigma$ may have mass at the origin. This shows that $\mu$ is a sequence of uniform distributions on spheres as indicated.

Remark. Since the mass points of a Nevanlinna extremal measure are the zeros of an entire function of exponential type, cf. $[1$, p. 56], we see that the radii of the corresponding spheres are the zeros of an entire function of order $\leqslant 2$.

\section{Measures of finite index}

In order to obtain more information about the measures in Theorem 4.4. we need the following

Lemma 5.1. Let $\sigma \in \mathcal{M}^{*}\left(\left[0, \infty[)\right.\right.$. If $t^{2} \operatorname{d\sigma }(t)$ is $\operatorname{det}(S)$ then $\sigma$ is determinate.

Proof. Let us assume that the measure $t^{2} d \sigma(t)$ is $\operatorname{det}(\mathrm{S})$. It has no atom at the origin, so by Proposition 1.1 it is also determinate. If $\tau \in \mathscr{M}^{*}(\mathbf{R})$ has the same moments 
as $\sigma$, then we have $t^{2} d \tau(t) \sim t^{2} d \sigma(t)$ and hence $t^{2} d \tau(t)=t^{2} \sigma(t)$. This show that $\tau=\sigma$, so $\sigma$ is determinate.

Proposition 5.2. The following properties of a measure $\sigma \in \mathcal{M}^{*}([0, \infty[)$ are equivalent:

(i) $\mathbf{C}[t]$ is dense in $L^{2}\left(t^{k} d \sigma(t)\right)$ for every $k \in \mathbf{N}$,

(ii) $t^{k} d \sigma(t)$ is $\operatorname{det}(S)$ for every $k \in \mathbf{N}$,

(iii) $t^{k} d \sigma(t)$ is determinate for every $k \in \mathbf{N}$,

(iv) $\mathbf{C}[t]$ is dense in $L^{2}\left(t^{p} d \sigma(t)\right)$ for every $p \in[0, \infty[$.

For a non-discrete measure $\sigma$ these properties are equivalent to $\mathbf{C}[t]$ being dense in $L^{2}(\sigma)$.

Proof. The implication "(i) $\Rightarrow$ (ii)" follows from Proposition 4.1, the implication "(ii) $\Rightarrow$ (iii)" follows from Lemma 5.1, and the implication "(iv) $\Rightarrow($ i)" is obvious. Suppose next that (iii) holds and let $p \in\left[0, \infty\left[\right.\right.$. If $k=[p]$ then $t^{p} \leqslant t^{k}\left(1+t^{2}\right)$ for $t \geqslant 0$, and by the theorem of Riesz $\mathbf{C}[t]$ is dense in $L^{2}\left(t^{k}\left(1+t^{2}\right) d \sigma(t)\right)$ and a fortiori in $L^{2}\left(t^{p} d \sigma(t)\right)$, so (iv) holds.

Suppose finally that $\sigma$ is non-discrete and that $\mathbf{C}[t]$ is dense in $L^{2}(\sigma)$. We claim that (i) holds. Assume the contrary. If $k \in \mathbf{N}$ is such that $\mathbf{C}[t]$ is dense in $L^{2}\left(t^{k} d \sigma(t)\right)$ but not dense in $L^{2}\left(t^{k+1} d \sigma(t)\right)$, then $t^{k} d \sigma(t)$ is indet(S) by Corollary 3.9 and hence Nevanlinna extremal. Therefore $t^{k} d \sigma(t)$ is discrete, which is a contradiction.

For a measure $\sigma \in \mathcal{M}^{*}([0, \infty[)$ which is $\operatorname{det}(\mathrm{S})$ we introduce the index (of determina$c y)$ of $\sigma$ as

$$
\operatorname{ind}(\sigma)=\sup \left\{k \in \mathbf{N} \mid t^{k} d \sigma(t) \text { is } \operatorname{det}(\mathrm{S})\right\} .
$$

The properties (i)-(iv) of Proposition 5.2 are equivalent to the statement ind $(\sigma)=\infty$. From Theorems 3.2, 3.6 and Proposition 5.2 we immediately get:

Proposition 5.3. Let $\mu \in \mathcal{M}_{\mathrm{rot}}^{*}\left(\mathbf{R}^{d}\right), d>1$, and put $\sigma=\mu^{\psi}$. The following statements are then equivalent:

(i) $\mu$ is determinate and the polynomials are not dense in $L^{2}(\mu)$.

(ii) $\sigma$ is $\operatorname{det}(S)$ and ind $(\sigma)<\infty$.

We shall characterize the class $\mathscr{S}$ defined in section 4 as the measures of index zero. As a preparation we need the following lemma.

LemMa 5.4. Assume that $\sigma \in \mathscr{M}^{*}([0, \infty[)$ has index zero, i.e. $\sigma$ is $\operatorname{det}(S)$ and $t \operatorname{d\sigma }(t)$ is indet $(S)$. Then $\sigma$ has no atom at the origin. 
Proof. The polynomials are dense in $L^{2}((1+t) d \sigma(t))$ by Theorem 3.8, because $\sigma$ is $\operatorname{det}(\mathbf{S})$. Assume that $\sigma$ has an atom at the origin. Then also $(1+t) d \sigma(t)$ has an atom at the origin, but if this mass is removed, the remaining measure is determinate by Corollary 1.5 , and so is the smaller measure $t d \sigma(t)$, in contradiction with the assumption.

THEOREM 5.5. A measure $v \in \mathcal{M}^{*}([0, \infty[)$ has index zero if and only if it belongs to $\mathscr{S}$. In the affirmative case $\mathrm{C}[t]$ is dense in $L^{2}\left(t^{k} d v(t)\right)$ for $k=0,1,2$, but not for any integer $k \geqslant 3$.

Proof. We have already remarked that a measure $\nu=\sigma_{0}-\sigma_{0}(\{0\}) \varepsilon_{0} \in \mathscr{S}$ is determinate and hence $\operatorname{det}(\mathrm{S})$. Since $t d v(t)=t d \sigma_{0}(t)$, we see that $t d v(t)$ is indet(S), so $v \in \mathscr{S}$ has index zero. We have also remarked that $\mathbf{C}[t]$ is dense in $L^{2}\left(t^{k} d v(t)\right)$ for $k=0,1,2$, but not for $k \geqslant 3$.

For the converse assume that $\nu \in \mathcal{M}^{*}([0, \infty[)$ has index zero. By Lemma 5.4 we get that $v$ has no atom at the origin, so $v$ is determinate by Proposition 1.1. Let $\alpha>0$ and put $\sigma=\alpha \varepsilon_{0}+v$. The measure $\sigma$ is then indet(S) and satisfies $\sigma=\sigma_{0}$ so that $v \in \mathscr{P}$. Indeed one has $t d \sigma(t)=t d v(t)$, which is indet(S). Thus, if $\sigma$ were $\operatorname{det}(\mathrm{S})$, it would have index zero and thereby have no atom at the origin by Lemma 5.4. We conclude that $\sigma$ is indet(S). Finally we have that $\mathbf{C}[t]$ is dense in $L^{2}(\sigma)$ by Corollary 1.5. This shows that $\sigma$ is Nevanlinna extremal with an atom at the origin and hence $\sigma=\sigma_{0}$.

ThEOREM 5.6. A measure $\sigma \in \mathcal{M}^{*}([0, \infty[)$ has finite index $\geqslant 1$ precisely when it is of the form

$$
\sigma=\alpha \varepsilon_{0}+t^{-j} d v(t)
$$

where $\alpha \geqslant 0, j$ is an integer $\geqslant 1$ and $v \in \mathscr{S}$.

In the affirmative case $\alpha=\sigma(\{0\}), j=\operatorname{ind}(\sigma)$ and $v=t^{\operatorname{ind}(\sigma)} d \sigma(t)$ so that the above representation is unique.

The polynomials are dense in $L^{2}\left(t^{k} d \sigma(t)\right)$ for $k=0,1, \ldots$, ind $(\sigma)+2$ but not for any integer $k \geqslant \operatorname{ind}(\sigma)+3$.

Proof. Assume that $\sigma \in \mathcal{M}^{*}([0, \infty[)$ has finite index $\geqslant 1$. If follows by Theorem 5.5 that $\nu=t^{\text {ind }(\sigma)} d \sigma(t)$ belongs to $\mathscr{S}$ so that the denseness assertion follows by Corollary 3.9 and Theorem 5.5, and $\sigma$ has the form indicated.

Conversely, if $\sigma$ has the form indicated, then $t^{j} d \sigma(t)=v$ is $\operatorname{det}(\mathrm{S})$. It follows that $\sigma$ is $\operatorname{det}(\mathrm{S})$, and since $t^{j+1} d \sigma(t)=t d v(t)$ is indet(S) because $\operatorname{ind}(v)=0$, we see that ind $(\sigma)=j$. 
Because of Proposition 5.3, the Theorems 5.5 and 5.6 show how to construct all measures $\mu \in \mathcal{M}_{\mathrm{rot}}^{*}\left(\mathbf{R}^{d}\right), d>1$, which are determinate and for which the polynomials are not dense in $L^{2}(\mu)$.

\section{Complements}

For a measure $\mu \in \mathcal{M}^{*}\left(\mathbf{R}^{d}\right)$ Fuglede [7] introduced the notions of strong determinacy and ultradeterminacy, and showed that the former is equivalent to $\mathbf{C}\left[x_{1}, \ldots, x_{d}\right]$ being dense in $L^{2}\left(\left(1+x_{j}^{2}\right) d \mu(x)\right)$ for each $j=1, \ldots, d$, while the latter is equivalent to $\mathbf{C}\left[x_{1}, \ldots, x_{d}\right]$ being dense in $L^{2}\left(\left(1+\|x\|^{2}\right) d \mu(x)\right)$. Fuglede noticed that ultradeterminacy of $\mu$ therefore implies strong determinacy which in turn implies determinacy and that the polynomials are dense in $L^{2}(\mu)$.

By Theorem 4.4 it is clear that there exist determinate $\mu \in \mathcal{M}_{\mathrm{rot}}^{*}\left(\mathbf{R}^{d}\right)$ which are not strongly determinate.

For another example of a determinate measure which is not strongly determinate see [15], which also contains an example of a strongly determinate measure which is not ultradeterminate.

For rotation invariant measures the two notions of strong determinacy and ultradeterminacy coincide, as we shall see below.

Let $s: \mathbf{N}^{d} \rightarrow \mathbf{R}$ be positive definite (cf. section 2). For polynomials $p(x)=\Sigma_{\alpha} c_{\alpha} x^{\alpha}$ and $q(x)=\Sigma_{\beta} d_{\beta} x^{\beta}$ we define their scalar product as

$$
\langle p, q\rangle:=\sum_{\alpha, \beta} c_{\alpha} \overline{d_{\beta}} s(\alpha+\beta)=L_{s}(p \tilde{q})
$$

By the Cauchy-Schwarz inequality the set $\mathcal{N}$ of polynomials $p$ with $\langle p, p\rangle=0$ is an ideal in $\mathbf{C}\left[x_{1}, \ldots, x_{d}\right]$. The quotient space $\mathbf{C}\left[x_{1}, \ldots, x_{d}\right] / \mathcal{N}$ is a pre-Hilbert space and we denote its completion by $\mathscr{P}$. The operator of multiplication with $x_{j}$ on $\mathbf{C}\left[x_{1}, \ldots, x_{d}\right]$ induces a densely defined symmetric operator $X_{j}$ in the Hilbert space $\mathscr{P}$ with domain $\mathbf{C}\left[x_{1}, \ldots, x_{d}\right] / \mathcal{N}$.

We shall say that $s$ satisfies Nelson's condition if the symmetric operator $X_{1}^{2}+\ldots+X_{d}^{2}$ is essentially self-adjoint. If $s$ satisfies Nelson's condition then a theorem of Nelson $[10$, p. $603 ; 12$, p. 489] together with a theorem of Fuglede [7, p. 57] imply that $s$ is the moment sequence of a strongly determinate measure. We shall say that a measure $\mu \in \mathcal{M}^{*}\left(\mathbf{R}^{d}\right)$ satisfies Nelson's condition if its moment sequence does. Fuglede [7, p. 59] noticed that Nelson's condition is equivalent to $\mathbf{C}\left[x_{1}, \ldots, x_{d}\right]$ being dense in $L^{2}\left(\left(1+\|x\|^{4}\right) d \mu(x)\right)$. In that case $\mu$ is even ultradeterminate because $1+\|x\|^{2} \leqslant 2\left(1+\|x\|^{4}\right)$. 
Proposition 6.1. The following properties of a measure $\mu \in \mathcal{M}_{\mathrm{rot}}^{*}\left(\mathbf{R}^{d}\right)$, where $d>1$, are equivalent:

(i) $\mathbf{C}\left[x_{1}, \ldots, x_{d}\right]$ is dense in $L^{2}(\mu)$,

(ii) $\mu$ satisfies Nelson's condition,

(iii) $\mu$ is ultradeterminate,

(iv) $\mu$ is strongly determinate.

Proof. Assume that (i) holds and let $\sigma=\mu^{\psi}$. According to Theorem 3.2, the polynomials are dense in $L^{2}\left(t^{k} d \sigma(t)\right)$ for every $k \in \mathbf{N}$. Proposition 5.2 then implies that $t^{k} d \sigma(t)$ is determinate for every $k \in \mathbf{N}$. But this means that $\mathbf{C}[t]$ is dense in $L^{2}\left(\left(1+t^{2}\right) t^{k} d \sigma(t)\right)$ for every $k \in \mathrm{N}$, cf. Proposition 1.3. This in turn implies by Theorem 3.2 that $\mathbf{C}\left[x_{1}, \ldots, x_{d}\right]$ is dense in $L^{2}\left(\left(1+\|x\|^{4}\right) d \mu(x)\right)$. The implications "(ii) $\Rightarrow$ (iii) $\Rightarrow$ (iv) $\Rightarrow$ (i)" are clear from the preceding discussion.

Remark. Assume that $\mu$ has the four equivalent properties of Proposition 6.1. Then any measure $v=h(x) d \mu(x)$ having a measurable and polynomially bounded density with respect to $\mu$ satisfies Nelson's condition.

To see this it suffices to show that the polynomials are dense in $L^{2}\left(\left(1+\|x\|^{4}\right)^{k} d \mu(x)\right)$ for any $k \in \mathbf{N}$. Assume the contrary and choose $k_{0} \in \mathbf{N}$ such that the polynomials are dense in $L^{2}\left(\left(1+\|x\|^{4}\right)^{k_{0}} d \mu(x)\right)$ but not dense in $L^{2}\left(\left(1+\|x\|^{4}\right)^{k_{0}+1} d \mu(x)\right)$. The measure $\tau=\left(1+\|x\|^{4}\right)^{k_{0}} d \mu(x)$ belongs to $\mathcal{M}_{\mathrm{rot}}^{*}\left(\mathbf{R}^{d}\right)$ and satisfies (i) but not (ii), which is a contradiction.

We shall give two examples showing that the converse of the first statements of Theorems 3.2 and 3.6 do not hold without symmetry in dimension one.

EXAMPLE 6.2. There exists a non-symmetric $\mu \in \mathcal{M}^{*}(\mathbf{R})$ such that $\mathbf{C}[x]$ is dense in $L^{2}(\mu)$ but not dense in $L^{2}\left(\mu^{\psi}\right)$.

Proof. Let $\nu \in \mathscr{S}$ and define $\sigma=t d v(t)$. Let $\tau \in \mathscr{M}^{*}(\mathbf{R})$ be the symmetric measure such that $\tau^{\psi}=\sigma$. Since $\sigma$ is indet(S) and $\mathbf{C}[t]$ is dense in $L^{2}(\sigma)$ and in $L^{2}(t d \sigma(t))$, cf. Theorem 5.5, we see that $\tau$ is indeterminate and Nevanlinna extremal, hence of the form

$$
\tau=\sum_{n=1}^{\infty} \alpha_{n}\left(\varepsilon_{a_{n}}+\varepsilon_{-a_{n}}\right)
$$

where $\alpha_{n}>0$ and $0<a_{1}<a_{2}<\cdots$. The measure

$$
\mu=\sum_{n=2}^{\infty} \alpha_{n}\left(\varepsilon_{a_{n}}+\varepsilon_{-a_{n}}\right)+2 \alpha_{1} \varepsilon_{a_{1}}+\varepsilon_{0}
$$


is again indeterminate and Nevanlinna extremal, cf. [2, section 4]. We have $\mu^{\psi}=\sigma+\varepsilon_{0}$, and since $\sigma$ is indeterminate, $\mathbf{C}[x]$ is not dense in $L^{2}\left(\mu^{\psi}\right)$ by Corollary 1.5.

EXAMPLE 6.3. There exists a non-symmetric and determinate $\mu_{1} \in \mathcal{M}^{*}(\mathbf{R})$ such that $\mu_{1}^{\psi}$ is indet $(S)$.

Proof. We define $\mu_{1}=\mu-\varepsilon_{0}$ with $\mu$ as in Example 6.2. Then $\mu_{1}$ is determinate by Proposition 1.4 and $\mu_{1}^{\psi}=\sigma$ is indet(S).

Acknowledgement. We wish to thank Bent Fuglede for valuable comments to the manuscript and in particular for having simplified an earlier version of Proposition 3.1.

\section{References}

[1] AkHIEzer, N. I., The Classical Moment Problem. Oliver and Boyd, Edinburgh, 1965.

[2] Berg, C. \& Christensen, J. P. R., Density questions in the classical theory of moments. Ann. Inst. Fourier, 31 (1981), 99-114.

[3] Berg, C., Christensen, J. P. R. \& Ressel, P., Harmonic Analysis on Semigroups. Theory of Positive Definite and Related Functions. Graduate Texts in Mathematics, Springer, Berlin - Heidelberg - New York, 1984.

[4] Bourbaki, N., Intégration. Herrmann, Paris, 1959.

[5] Buchwalter, H \& Cassier, G., La paramétrisation de Nevanlinna dans le problème des moments de Hamburger. Exposition. Math., 2 (1984), 155-178.

[6] Chinara, T. S., Indeterminate symmetric moment problems. J. Math. Anal. Appl., 85 (1982), 331-346.

[7] Fuglede, B., The multidimensional moment problem. Exposition. Math., 1 (1983), 47-65.

[8] HAVIN, V. P. ET AL. (editors), Linear and complex analysis problem book. 199 research problems. Lecture Notes in Mathematics, 1043. Springer, Berlin-Heidelberg-New York, 1984.

[9] Heyde, C. C., Some remarks on the moment problem I. Quart. J. Math. Oxford (2), 14 (1963), 91-96.

[10] Nelson, E., Analytic vectors. Ann. Math. (2), 70 (1957), 572-615.

[11] Nevanlinna, R., Asymptotische Entwicklungen beschränkter Funktionen und das Stieltjessche Momentenproblem. Ann. Acad. Sci. Fenn. Ser. AI, 18:5 (1922), (52 pp.).

[12] Nussbaum, A. E., A commutativity theorem for unbounded operators in Hilbert space. Trans. Amer. Math. Soc., 140 (1969), 485-491.

[13] Petersen, L. C., On the relation between the multidimensional moment problem and the one-dimensional moment problem. Math. Scand., 51 (1982), 361-366.

[14] Riesz, M., Sur le problème des moments et le théorème de Parseval correspondant. Acta Litt. Ac. Sci. Szeged, 1 (1923), 209-225.

[15] SchmÜdgen, K., On determinacy notions for the two dimensional moment problem. To appear in Ark. Mat.

[16] SteIN, E. M. \& WeIss, G., Introduction to Fourier Analysis on Euclidean Spaces. Princeton University Press, Princeton, 1971.

Received April 3, 1990

Received in revised form August 20, 1990 